\title{
Article \\ Some Geometric Constants Related to the Midline of Equilateral Triangles in Banach Spaces
}

\author{
Bingren Chen (1), Zhijian Yang, Qi Liu (1) and Yongjin Li *(]) \\ School of Mathematics, Sun Yat-sen University, Guangzhou 510275, China; chenbr3@mail2.sysu.edu.cn (B.C.); \\ yangzhj55@mail2.sysu.edu.cn (Z.Y.); liuq325@mail2.sysu.edu.cn (Q.L.) \\ * Correspondence: stslyj@mail.sysu.edu.cn
}

Citation: Chen, B.; Yang, Z.; Liu, Q.; Li, Y. Some Geometric Constants

Related to the Midline of Equilateral Triangles in Banach Spaces. Symmetry 2022, 14, 348. https://doi.org/ $10.3390 /$ sym 14020348

Academic Editor: Roman Ger

Received: 25 January 2022

Accepted: 7 February 2022

Published: 9 February 2022

Publisher's Note: MDPI stays neutral with regard to jurisdictional claims in published maps and institutional affiliations.

Copyright: (C) 2022 by the authors. Licensee MDPI, Basel, Switzerland. This article is an open access article distributed under the terms and conditions of the Creative Commons Attribution (CC BY) license (https:// creativecommons.org/licenses/by/ $4.0 /)$.

\begin{abstract}
We will introduce some new geometric constants based on the constant $H(X)$ proposed by Gao and the constant $A_{2}(X)$ proposed by M. Baronti et al. We first provide a study of a new constant $M_{1}(X)$ closely related to the midlines of equilateral triangles, including a discussion of some of its properties and the connections with other parameters of the sphere. Next, we focus on a new constant $M_{2}(X)$ and its generalized form $M_{2}(X, p, q)$, along with some of their basic properties. Finally, we concentrate on a new constant $M_{3}(X)$ and discuss some of its properties.
\end{abstract}

Keywords: Banach spaces; geometric constants; uniformly non-square

\section{Introduction}

Recently, many investigations have been devoted to geometric constants of a Banach space $X$, among which, the Jordan-von Neumann constant $C_{N J}(X)$ and the James constant $J(X)$ have been widely studied, and their results have greatly motivated us to make specific descriptions of a variety of geometric properties of $X$.

Recall a classic constant $A_{2}(X)$ proposed by M. Baronti, E. Casini, P.L. Papini in 2000 [1] that manifested antipodal points $y$ and $-y$ on the unit sphere $S_{X}$, where $\|x+y\|,\|x-y\|$ and 2 could be considered as the lengths of the sides of the triangle with vertices $x, y,-y$ lying on $S_{X}$. It is noteworthy that the constant $A_{2}(X)$ describes the supremum of the arithmetic mean of the lengths of the changeable sides of the triangle, i.e.,

$$
A_{2}(X)=\sup \left\{\frac{\|x+y\|+\|x-y\|}{2}:\|x\|=\|y\|=1\right\} .
$$

Alonso and Llorens-Fuster [2] have discussed the relation between $J(X)$ and $A_{2}(X)$ as

$$
A_{2}(X) \leq 1+\frac{J(X)}{2}
$$

Strongly motivated by the asymmetric constant $H(X)$ proposed by Gao in 2000 [3], Chen et al. has further investigated the constant $H(X)$ proposed by Gao by discussing several properties of it that have not yet been discovered. A constant $G_{L}(X)$ closely relevant to $H(X)$, along with a variety of geometric properties and several relations among it and several basic geometric constants, has also been shown via a few inequalities. For more details about the aforementioned results, we recommend reference [4].

In this paper, we intend to conduct further research on the related constants combined with the Baronti constant. Firstly, we mainly focus on a new Baronti type constant $M_{1}(X)$ in Section 3, which combines the unit sphere of the Banach spaces with the inscribed equilateral triangles and innovatively takes the quantity $2 x-y$ into consideration. Then, we want to bring out a few connections among its value and some geometrical properties of the space. Additionally, the relations among $M_{1}(X)$ and some other constants such as $H(X)$ will be revealed by the clarification of several inequalities. $M_{1}(X)$ can measure how 
large the sum of two distances from a point of the inscribed equilateral triangle to the midpoint of the opposite side can be. In other terms, their values hinges on the lengths of two midlines of a triangle with one vertex at the center of the unit ball and the other on the unit sphere. Moreover, some inequalities enable us to continue introducing some other new constants $M_{2}(X), M_{2}(X, p, q)$ and $M_{3}(X)$. It is interesting to study the non-symmetrical properties of these constants and hopefully obtain some significant results.

\section{Preliminaries}

Throughout the paper, let $X$ be a non-trivial Banach space, that is, $\operatorname{dim} X \geq 2$, and use $S_{X}$ and $B_{X}$ to represent the unit sphere and closed unit ball of $X$, respectively.

Definition 1. A Banach space $X$ is called uniformly non-square if there exists $\delta \in(0,1)$ such that for any $x, y \in S_{X}$, we have either $\frac{\|x+y\|}{2} \leq 1-\delta$ or $\frac{\|x-y\|}{2} \leq 1-\delta$.

The geometric constant below was proposed by Yang et al. [5]:

Definition 2. Let $X$ be a Banach space. The function $\gamma_{X}(t):[0,1] \rightarrow[0,4]$ is defined by

$$
\gamma_{X}(t)=\sup \left\{\frac{\|x+t y\|^{2}+\|x-t y\|^{2}}{2}: x, y \in S_{X}\right\} .
$$

Clearly the following assertion was also shown in the paper [5]:

$$
\gamma_{X}(t)=\sup \left\{\frac{\|x+t y\|^{2}+\|x-t y\|^{2}}{2}: x, y \in B_{X}\right\} .
$$

J. Lindenstrauss [6] has studied several properties of the following modulus of smoothness of $X$.

Definition 3. Let X be a Banach space. The modulus of smoothness is defined by

$$
\rho_{X}(t)=\sup \left\{\frac{\|x+t y\|+\|x-t y\|}{2}-1: x, y \in B_{X}\right\} .
$$

$X$ is called uniformly smooth if $\lim _{t \rightarrow 0} \frac{\rho_{X}(t)}{t}=0$.

In addition, we recall the definitions of some main geometric constants as follows.

\section{Definition 4.}

(i) The James constant (Gao [7], Gao and Lau [8]):

$$
J(X):=\sup \{\min (\|x+y\|,\|x-y\|):\|x\|=\|y\|=1\} ;
$$

(ii) The Jordan-Von Neumann constant (Clarkson [9]):

$$
\begin{aligned}
C_{N J}(X): & =\sup \left\{\frac{\|x+y\|^{2}+\|x-y\|^{2}}{2\left(\|x\|^{2}+\|y\|^{2}\right)}: x, y \in X,\|x\|+\|y\| \neq 0\right\} \\
& =\sup \left\{\frac{\|x+y\|^{2}+\|x-y\|^{2}}{2\left(\|x\|^{2}+\|y\|^{2}\right)}: x \in S_{X}, y \in B_{X}\right\} ;
\end{aligned}
$$

(iii) The hexagon constant (Gao [3]):

$$
H(X):=\sup \left\{\min \{\|x+y\|,\|2 x-y\|\}: x, y, x-y \in S_{X}\right\} ;
$$


(iv) The Baronti constant (Baronti et al. [1]):

$$
A_{2}(X):=\sup \left\{\frac{\|x+y\|+\|x-y\|}{2}:\|x\|=\|y\|=1\right\} .
$$

Now we enumerate several results of the aforementioned constants. Gao has conducted a study of $H(X)$ with the following properties in 2000 [3]:

(i) For a Banach space, $H(X) \leq 2$;

(ii) If $X$ is a Hilbert space, then $H(X)=\sqrt{3}$;

(iii) Let $X=\ell_{p}$ or $L_{p} \equiv L_{p}[0,1]$. Let $1<p<\infty$ be defined as usual. Then, $H(X) \leq$ $\left(2^{p}-1\right)^{\frac{1}{p}}$ for $p \geq 2 ; H(X) \leq 3^{\frac{1}{p}}$ for $1 \leq p<2$;

(iv) If $H(X)<2$, then $X$ is uniformly non-square.

Investigations on the relations among $J(X), A_{2}(X)$ and $C_{N J}(X)$ can be found in [1,10-12], along with their common geometric properties.

(i) $\sqrt{2} \leq J(X) \leq A_{2}(X) \leq 2,1 \leq C_{N J}(X) \leq 2, J(X)^{2} \leq A_{2}(X)^{2} \leq C_{N J}(X)$;

(ii) If $X$ is a Hilbert space, then $J(X)=\sqrt{2}$, a fortiori $A_{2}(X)=\sqrt{2}$;

(iii) $X$ is uniformly non-square if and only if one of the following is true: (a) $J(X)<2$, (b) $C_{N J}(X)<2,(\mathrm{c}) A_{2}(X)<2$.

\section{Some Inequalities Related to New Constant $M_{1}(X)$}

The heuristic idea in this paper comes from a study of the height lines of triangle, which were defined as the height vector $h(x, y)$, and then provided to give the new characterizations of inner product spaces [13]. Highly inspired by the study of height lines of triangle and its discussion of properties in real normed space, we will explore the properties of midlines of triangle via discussion of the new constant $M_{1}(X)$ along with its properties. Firstly, we define the constant $M_{1}(X)$ of a Banach space $X$ as follows:

\section{Definition 5.}

$$
M_{1}(X)=\sup \left\{\frac{\|x+y\|+\|2 x-y\|}{2}:\|x\|=\|y\|=\|x-y\|=1\right\} .
$$

The geometric background of $M_{1}(X)$ is shown in Figure 1: consider the unit sphere in the Euclidean plane with $\|x\|=\|y\|=\|y-x\|=1$. Assume that $\overrightarrow{O A}=x, \overrightarrow{O B}=y$. Then, $\overrightarrow{B A}=x-y$. Assume that $C$ is the midpoint of $\overrightarrow{O B}$, and $D$ is the midpoint of $\overrightarrow{B A}=x-y$. Then, $\overrightarrow{C A}=x-\frac{y}{2}$ and $\overrightarrow{O D}=\frac{x+y}{2}$. Apparently, the geometric concept of $M_{1}(X)$ is the supremum of sum of two midlines.

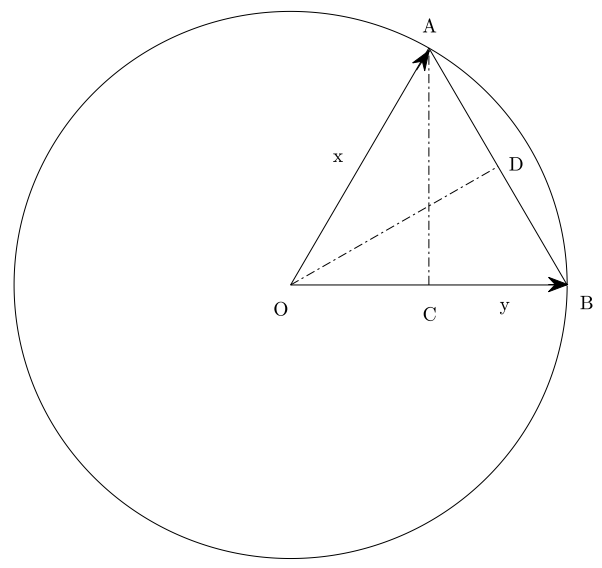

Figure 1. Geometric explanation on the equilateral triangle $\triangle O A B$. 
Proposition 1. If $X$ is an inner product space, then $M_{1}(X)=\sqrt{3}$.

Proof. For any $x, y, x-y \in S_{X}$,

$$
\|x+y\|^{2}=2\left(\|x\|^{2}+\|y\|^{2}\right)-\|x-y\|^{2}=3,
$$

and

$$
\|2 x-y\|^{2}=2\left(\|x\|^{2}+\|x-y\|^{2}\right)-\|x-(x-y)\|^{2}=3 .
$$

Therefore, $M_{1}(X)=\sup \left\{\frac{\|x+y\|+\|2 x-y\|}{2}:\|x\|=\|y\|=\|x-y\|=1\right\}=\sqrt{3}$.

Proposition 2. Let $X$ be a finite-dimensional Banach space. If $M_{1}(X)=2$, then $X$ is not rotund.

Proof. Since $X$ is a finite-dimensional Banach space with $M_{1}(X)=2$, there exist $x_{0}, y_{0}, x_{0}-$ $y_{0} \in S_{X}$, such that

$$
\frac{\left\|x_{0}+y_{0}\right\|+\left\|2 x_{0}-y_{0}\right\|}{2}=2 \text {, }
$$

so

$$
\left\|x_{0}+y_{0}\right\|+\left\|2 x_{0}-y_{0}\right\|=4
$$

thus

$$
4=\left\|x_{0}+y_{0}\right\|+\left\|2 x_{0}-y_{0}\right\| \leq\left\|x_{0}\right\|+\left\|y_{0}\right\|+\left\|x_{0}\right\|+\left\|x_{0}-y_{0}\right\|=4
$$

hence

$$
\left\|x_{0}+y_{0}\right\|+\left\|2 x_{0}-y_{0}\right\|=\left\|x_{0}\right\|+\left\|y_{0}\right\|+\left\|x_{0}\right\|+\left\|x_{0}-y_{0}\right\|=4 \text {. }
$$

Combining with $\left\|x_{0}+y_{0}\right\| \leq\left\|x_{0}\right\|+\left\|y_{0}\right\|=2,\left\|2 x_{0}-y_{0}\right\| \leq\left\|x_{0}\right\|+\left\|x_{0}-y_{0}\right\|=2$, we have $\left\|x_{0}+y_{0}\right\|=\left\|2 x_{0}-y_{0}\right\|=2$. By $\left\|x_{0}\right\|=\left\|y_{0}\right\|=1$ and $\left\|x_{0}+y_{0}\right\|=2$; therefore, we obtain that $X$ is not rotund.

Proposition 3. Let $X$ be Banach space. Then $M_{1}(X) \leq \frac{3}{2} \rho_{X}\left(\frac{2}{3}\right)+\frac{3}{2}$.

Proof. Note that

$$
\begin{aligned}
\frac{\|x+y\|+\|2 x-y\|}{2} & =\frac{\left\|\frac{3}{2} x-\left(\frac{1}{2} x-y\right)\right\|+\left\|\frac{3}{2} x+\left(\frac{1}{2} x-y\right)\right\|}{2} \\
& =\frac{3}{4}\left(\left\|x-\frac{1}{3}(x-2 y)\right\|+\left\|x+\frac{1}{3}(x-2 y)\right\|\right) \\
& =\frac{3}{4}\left(\left\|x-\frac{2}{3} \cdot \frac{x-2 y}{2}\right\|+\left\|x+\frac{2}{3} \cdot \frac{x-2 y}{2}\right\|\right) .
\end{aligned}
$$

So

We can let $x, y, x-y \in S_{X}$, and deduce $\frac{1}{2} \leq\left\|\frac{x-2 y}{2}\right\| \leq 1$, which implies that $\frac{x-2 y}{2} \in B_{X}$.

$$
\frac{\|x+y\|+\|2 x-y\|}{2} \leq \frac{3}{2} \rho_{X}\left(\frac{2}{3}\right)+\frac{3}{2},
$$

that is, $M_{1}(X) \leq \frac{3}{2} \rho_{X}\left(\frac{2}{3}\right)+\frac{3}{2}$.

Proposition 4. Let $X$ be a Banach space. Then $M_{1}(X) \leq \frac{3}{2} \sqrt{\gamma_{X}\left(\frac{2}{3}\right)}$. 
Proof. Note that

$$
\begin{aligned}
\frac{\|x+y\|+\|2 x-y\|}{2} & =\frac{\left\|\frac{3}{2} x-\left(\frac{1}{2} x-y\right)\right\|+\left\|\frac{3}{2} x+\left(\frac{1}{2} x-y\right)\right\|}{2} \\
& =\frac{3}{4}\left(\left\|x-\frac{1}{3}(x-2 y)\right\|+\left\|x+\frac{1}{3}(x-2 y)\right\|\right) \\
& =\frac{3}{4}\left(\left\|x-\frac{2}{3} \cdot \frac{x-2 y}{2}\right\|+\left\|x+\frac{2}{3} \cdot \frac{x-2 y}{2}\right\|\right) \\
& \leq \frac{3}{2} \sqrt{\frac{\left\|x-\frac{2}{3} \cdot \frac{x-2 y}{2}\right\|^{2}+\left\|x+\frac{2}{3} \cdot \frac{x-2 y}{2}\right\|^{2}}{2}} .
\end{aligned}
$$

We can let $x, y, x-y \in S_{X}$, and deduce that

$$
\frac{1}{2} \leq\left\|\frac{x-2 y}{2}\right\| \leq 1
$$

which implies that $\frac{x-2 y}{2} \in B_{X}$. Therefore,

$$
\frac{\|x+y\|+\|2 x-y\|}{2} \leq \frac{3}{2} \sqrt{\gamma_{X}\left(\frac{2}{3}\right)}
$$

that is, $M_{1}(X) \leq \frac{3}{2} \sqrt{\gamma_{X}\left(\frac{2}{3}\right)}$.

Proposition 5. For any Banach space X,

$$
H(X) \leq M_{1}(X) \leq \frac{H(X)}{2}+1 .
$$

Proof. For $x, y, x-y \in S_{X}$, we have

$$
\begin{aligned}
\|x+y\|+\|2 x-y\| & =\min \{\|x+y\|,\|2 x-y\|\}+\max \{\|x+y\|,\|2 x-y\|\} \\
& \leq \min \{\|x+y\|,\|2 x-y\|\}+2 \\
& \leq H(X)+2
\end{aligned}
$$

which gives $M_{1}(X) \leq \frac{H(X)}{2}+1$.

Since

$$
\min \{\|x+y\|,\|2 x-y\|\} \leq \frac{\|x+y\|+\|2 x-y\|}{2},
$$

we obtain the left-hand side of the inequality.

Theorem 1. Let $X$ be a Banach space. If $M_{1}(X)<2$, then $X$ is uniformly non-square.

Proof. Applying Proposition 5, we have $H(X)<2$. Utilizing the result in ([3], p. 243, Theorem 2.10), then $X$ is uniformly non-square.

Corollary 1. Let $X$ be a Banach space. If $M_{1}(X)<2$, then $X$ has the fixed point property.

Proof. Utilizing Theorem 1 and [14], every uniformly non-square Banach space has the fixed point property, so we get the result as required.

Proposition 6. Let $X$ be a Banach space. Then $\frac{3}{2} \leq M_{1}(X) \leq 2$. 
Proof. For any $x, y, x-y \in S_{X}$, we have

$$
\|x+y\|+\|2 x-y\| \geq\|(x+y)+(2 x-y)\|=3,
$$

and then $M_{1}(X) \geq \frac{3}{2}$.

$$
\|x+y\|+\|2 x-y\| \leq\|x\|+\|y\|+\|x\|+\|x-y\|=4,
$$

and so $M_{1}(X) \leq 2$.

Example 1. Let $X=\ell_{\infty}$ endowed with the norm $\|x\|_{\infty}=\sup _{n}\left|x_{n}\right|$ for $\left(x_{n}\right) \in \ell_{\infty}$. Then, $M_{1}\left(\ell_{\infty}\right)=2$. $S\left(\ell_{\infty}\right)$.

Assume that $x=(1,1,0, \ldots), y=(1,0,0, \ldots) \in S\left(\ell_{\infty}\right) ;$ then, $x-y=(0,1,0, \ldots) \in$

Then, $M_{1}\left(\ell_{\infty}\right) \geq \frac{\|x+y\|+\|2 x-y\|}{2}=2$. Since $M_{1}\left(\ell_{\infty}\right) \leq 2$, so $M_{1}\left(\ell_{\infty}\right)=2$.

Example 2. Let $X$ be $\mathbb{R}^{2}$ endowed with the $\ell_{\infty}-\ell_{1}$ norm defined by

$$
\|x\|=\left\|\left(x_{1}, x_{2}\right)\right\|= \begin{cases}\max \left\{\left|x_{1}\right|,\left|x_{2}\right|\right\}, & x_{1} x_{2} \geq 0 \\ \left|x_{1}\right|+\left|x_{2}\right|, & x_{1} x_{2}<0\end{cases}
$$

Assume that $x=(1,1), y=(1,0) \in S_{X}$; then $x-y=(0,1) \in S_{X}$. Then,

$$
M_{1}(X) \geq \frac{\|x+y\|+\|2 x-y\|}{2}=2,
$$

and then we have

$$
M_{1}(X) \leq \frac{\|x\|+\|y\|+\|x\|+\|x-y\|}{2}=2
$$

Therefore,

$$
M_{1}(X)=2
$$

\section{Some Results Related to New Constants $M_{2}(X)$ and $M_{3}(X)$}

For any Banach space $X$, we define another constant $M_{2}(X)$, which may be different from $M_{1}(X)$ in view of its value. We also want to bring out the relations among $M_{2}(X)$ and some geometric constants, along with its geometric properties.

\section{Definition 6.}

$$
M_{2}(X)=\sup \left\{\frac{\|x+y\|^{2}+\|2 x-y\|^{2}}{2}:\|x\|=\|y\|=\|x-y\|=1\right\} .
$$

Proposition 7. If $X$ is an inner product space, then $M_{2}(X)=3$.

Proof. For any $x, y, x-y \in S_{X}$,

$$
\|x+y\|^{2}=2\left(\|x\|^{2}+\|y\|^{2}\right)-\|x-y\|^{2}=3,
$$

and

$$
\begin{gathered}
\|2 x-y\|^{2}=2\left(\|x\|^{2}+\|x-y\|^{2}\right)-\|x-(x-y)\|^{2}=3 . \\
\text { Therefore, } M_{2}(X)=\sup \left\{\frac{\|x+y\|^{2}+\|2 x-y\|^{2}}{2}:\|x\|=\|y\|=\|x-y\|=1\right\}=3 .
\end{gathered}
$$


Proposition 8. For any Banach space X,

$$
\left(M_{1}(X)\right)^{2} \leq M_{2}(X) \leq \frac{(H(X))^{2}}{2}+2 .
$$

Proof. For $x, y, x-y \in S_{X}$, we have

$$
\begin{aligned}
\|x+y\|^{2}+\|2 x-y\|^{2} & =\min \left\{\|x+y\|^{2},\|2 x-y\|^{2}\right\}+\max \left\{\|x+y\|^{2},\|2 x-y\|^{2}\right\} \\
& \leq \min \left\{\|x+y\|^{2},\|2 x-y\|^{2}\right\}+4 \\
& \leq(H(X))^{2}+4
\end{aligned}
$$

which gives $M_{2}(X) \leq \frac{(H(X))^{2}}{2}+2$.

Since $(\|x+y\|+\|2 x-y\|)^{2} \leq 2\left(\|x+y\|^{2}+\|2 x-y\|^{2}\right)$, we obtain the left-hand side of the inequality.

Theorem 2. Let $X$ be a Banach space. If $M_{2}(X)<4$, then $X$ is uniformly non-square.

Proof. Applying Proposition 8 , we have $M_{1}(X)<2$. Utilizing Theorem 1 , then $X$ is uniformly non-square.

Next, we consider the relation between $M_{2}(X)$ and $\gamma_{X}(t)$ through inequality.

Theorem 3. For any Banach space X,

$$
M_{2}(X) \leq 1+2 \gamma_{X}(1)
$$

Proof. For any $x, y, x-y \in S_{X}$, we have

$$
\begin{aligned}
\frac{\|x+y\|^{2}+\|2 x-y\|^{2}}{2} & \leq \frac{\|x+y\|^{2}+(\|x\|+\|x-y\|)^{2}}{2} \\
& \leq \frac{\|x+y\|^{2}+2\|x\|^{2}+2\|x-y\|^{2}}{2} \\
& \leq 1+\frac{2\|x+y\|^{2}+2\|x-y\|^{2}}{2} \\
& \leq 1+2 \gamma_{X}(1) .
\end{aligned}
$$

Therefore, we complete the proof.

Additionally, for the sake of further survey on the upper bounds of $M_{2}(X)$, we utilize a parameter $E(X)$, which is defined as follows:

$$
E(X)=\sup \left\{\|x+y\|^{2}+\|x-y\|^{2}:\|x\|=\|y\|=1\right\} .
$$

Theorem 4. For any Banach space X,

$$
M_{2}(X) \geq \frac{E(X)}{2}-\frac{1}{2} .
$$


Proof. For any $x, y, x-y \in S_{X}$, we have

$$
\begin{aligned}
\frac{\|x+y\|^{2}+\|2 x-y\|^{2}}{2} & \geq \frac{\|x+y\|^{2}+\left(\|x-y\|^{2}\right.}{2} \\
& =\frac{\|x+y\|^{2}+\|x-y\|^{2}-2\|x-y\|+1}{2} \\
& =\frac{\|x+y\|^{2}+\|x-y\|^{2}}{2}-\|x-y\|+\frac{1}{2} \\
& =\frac{\|x+y\|^{2}+\|x-y\|^{2}}{2}-\frac{1}{2} .
\end{aligned}
$$

Thus, we complete the proof.

Theorem 5. For any Banach space $X$, if $M_{2}(X)=4$, then $H(X)=2$.

Proof. For any $\varepsilon>0$, there exist $x_{\varepsilon}, y_{\varepsilon} \in S_{X}$ such that

$$
\frac{\left\|x_{\varepsilon}+y_{\varepsilon}\right\|^{2}+\left\|2 x_{\varepsilon}-y_{\varepsilon}\right\|^{2}}{2}>4-\varepsilon \text {. }
$$

Thus

$$
\left\|x_{\varepsilon}+y_{\varepsilon}\right\|^{2}+\left\|2 x_{\varepsilon}-y_{\varepsilon}\right\|^{2}>8-2 \varepsilon \text {. }
$$

Since $\left\|x_{\varepsilon}+y_{\varepsilon}\right\| \leq 2$, and $\left\|2 x_{\varepsilon}-y_{\varepsilon}\right\| \leq\left\|x_{\varepsilon}\right\|+\left\|x_{\varepsilon}-y_{\varepsilon}\right\|=2$, it can be deduced that

$$
4+\min \left\{\left\|x_{\varepsilon}+y_{\varepsilon}\right\|^{2},\left\|2 x_{\varepsilon}-y_{\varepsilon}\right\|^{2}\right\} \geq 8-2 \varepsilon
$$

Therefore, we obtain

$$
\left\|x_{\varepsilon}+y_{\varepsilon}\right\|>\sqrt{4-2 \varepsilon}, \quad\left\|2 x_{\varepsilon}-y_{\varepsilon}\right\|>\sqrt{4-2 \varepsilon} .
$$

A fortiori, we have

$$
H(X) \geq \min \left\{\left\|x_{\varepsilon}+y_{\varepsilon}\right\|,\left\|2 x_{\varepsilon}-y_{\varepsilon}\right\|\right\}>\sqrt{4-2 \varepsilon} .
$$

Now that $\varepsilon$ can be arbitrarily small, then

$$
H(X) \geq 2
$$

Combined with the fact that

$$
H(X) \leq 2
$$

we have $H(X)=2$.

Now we shall consider the generalised form of $M_{2}(X)$, which is defined as follows.

\section{Definition 7.}

$$
M_{2}(X, p, q)=\sup \left\{\frac{1}{2}\|x+y\|^{p}+\frac{1}{2}\|2 x-y\|^{q}:\|x\|=\|y\|=\|x-y\|=1\right\} .
$$

Proposition 9. If $X$ is an inner space, then $M_{2}(X, p, q)=\frac{3^{\frac{p}{2}}+3^{\frac{q}{2}}}{2}$.

Proof. For any $x, y, x-y \in S_{X}$

$$
\|x+y\|^{2}=2\left(\|x\|^{2}+\|y\|^{2}\right)-\|x-y\|^{2}=3,
$$


and

$$
\|2 x-y\|^{2}=2\left(\|x\|^{2}+\|x-y\|^{2}\right)-\|x-(x-y)\|^{2}=3 .
$$

Thus, $M_{2}(X, p, q)=\sup \left\{\frac{1}{2}\|x+y\|^{p}+\frac{1}{2}\|2 x-y\|^{q}:\|x\|=\|y\|=\|x-y\|=1\right\}=$ $\frac{3^{\frac{p}{2}}+3^{\frac{q}{2}}}{2}$

Example 3. Let $X$ be $\ell_{p}, 1 \leq p<2 \leq q<\infty$. Then, $M_{2}(X, p, q) \leq 2^{1+\frac{q}{p}}+2$.

Applying Clarkson's type inequality, when $1 \leq p<2, x, y \in X$, we have

$$
\begin{aligned}
2\left(\|x\|_{p}^{p}+\|y\|_{p}^{p}\right) & \geq\|x+y\|_{p}^{p}+\|x-y\|_{p}^{p} \\
& \geq\left(\|x\|_{p}+\|y\|_{p}\right)^{p}+\left|\|x\|_{p}-\|y\|_{p}\right|^{p} .
\end{aligned}
$$

We also use the following Clarkson's inequality

$$
\|x+y\|_{p}^{q}+\|x-y\|_{p}^{q} \leq 2\left(\|x\|_{p}^{p}+\|y\|_{p}^{p}\right)^{\frac{q}{p}} .
$$

Then we can deduce that for any $x, y, x-y \in S_{X}$,

$$
\begin{aligned}
\|x+y\|_{p}^{p} & \leq 2\left(\|x\|_{p}^{p}+\|y\|_{p}^{p}\right)-\|x-y\|_{p}^{p} \\
& \leq 2\left(\|x\|_{p}^{2}+\|y\|_{p}^{2}\right)-\|x-y\|_{p}^{2} \\
& =4-1 \\
& =3,
\end{aligned}
$$

$$
\begin{aligned}
\|2 x-y\|_{p}^{q} & \leq 2\left(\|x\|_{p}^{p}+\|x-y\|_{p}^{p}\right)^{\frac{q}{p}}-\|x-(x-y)\|_{p}^{q} \\
& \leq 2^{1+\frac{q}{p}}-1,
\end{aligned}
$$

as desired.

Example 4. Let $X$ be $\mathbb{R}^{2}$ endowed with the $\ell_{\infty}-\ell_{1}$ norm defined by

$$
\|x\|=\left\|\left(x_{1}, x_{2}\right)\right\|= \begin{cases}\max \left\{\left|x_{1}\right|,\left|x_{2}\right|\right\}, & x_{1} x_{2} \geq 0 \\ \left|x_{1}\right|+\left|x_{2}\right|, & x_{1} x_{2}<0\end{cases}
$$

Assume that $x=(1,1), y=(1,0) \in S_{X}$; then $x-y=(0,1) \in S_{X}$. Then,

$$
M_{2}\left(\ell_{\infty}-\ell_{1}\right) \geq \frac{\|x+y\|^{2}+\|2 x-y\|^{2}}{2}=4,
$$

and we thus have

$$
M_{2}\left(\ell_{\infty}-\ell_{1}\right) \leq \frac{(\|x\|+\|y\|)^{2}+(\|x\|+\|x-y\|)^{2}}{2}=4,
$$

Therefore,

$$
\begin{gathered}
M_{2}\left(\ell_{\infty}-\ell_{1}\right)=4 . \\
M_{2}\left(\ell_{\infty}-\ell_{1}, p, q\right) \geq \frac{1}{2}\|x+y\|^{p}+\frac{1}{2}\|2 x-y\|^{q}=2^{p-1}+2^{q-1},
\end{gathered}
$$

and then we have

$$
M_{2}\left(\ell_{\infty}-\ell_{1}, p, q\right) \leq \frac{1}{2}(\|x\|+\|y\|)^{p}+\frac{1}{2}(\|x\|+\|x-y\|)^{q}=2^{p-1}+2^{q-1},
$$


Therefore

$$
M_{2}\left(\ell_{\infty}-\ell_{1}, p, q\right)=2^{p-1}+2^{q-1} .
$$

Note that the values of the subduplicate form of the aforementioned constants can also be different. We therefore consider another new constant $M_{3}(X)$, which is denoted as follows:

\section{Definition 8.}

$$
M_{3}(X)=\sup \left\{\frac{\|x+y\|^{\frac{1}{2}}+\|2 x-y\|^{\frac{1}{2}}}{2}:\|x\|=\|y\|=\|x-y\|=1\right\} .
$$

Proposition 10. If $X$ is an inner product space, then $M_{3}(X)=3^{\frac{1}{4}}$.

Proof. For any $x, y, x-y \in S_{X}$,

$$
\|x+y\|^{2}=2\left(\|x\|^{2}+\|y\|^{2}\right)-\|x-y\|^{2}=3,
$$

and

$$
\|2 x-y\|^{2}=2\left(\|x\|^{2}+\|x-y\|^{2}\right)-\|x-(x-y)\|^{2}=3 .
$$

Therefore $M_{3}(X)=\sup \left\{\frac{\|x+y\|^{\frac{1}{2}}+\|2 x-y\|^{\frac{1}{2}}}{2}:\|x\|=\|y\|=\|x-y\|=1\right\}=3^{\frac{1}{4}}$.

Proposition 11. For any Banach space X,

$$
(H(X))^{\frac{1}{2}} \leq M_{3}(X) \leq\left(M_{1}(X)\right)^{\frac{1}{2}} .
$$

Proof. Since $x^{\frac{1}{2}}$ is concave, then for any $x, y, x-y \in S_{X}$, we have

$$
\frac{\|x+y\|^{\frac{1}{2}}+\|2 x-y\|^{\frac{1}{2}}}{2} \leq\left(\frac{\|x+y\|+\|2 x-y\|}{2}\right)^{\frac{1}{2}},
$$

which gives $M_{3}(X) \leq\left(M_{1}(X)\right)^{\frac{1}{2}}$.

Since $\min \{\|x+y\|,\|2 x-y\|\}^{\frac{1}{2}} \leq \frac{\|x+y\|^{\frac{1}{2}}+\|2 x-y\|^{\frac{1}{2}}}{2}$, we obtain the left-hand side of the inequality.

Theorem 6. Let $X$ be a Banach space. If $M_{3}(X)<\sqrt{2}$, then $X$ is uniformly non-square.

Proof. Applying Proposition 11, we have $H(X)<2$. Utilizing the result in ([3], p. 243, Theorem 2.10), then $X$ is uniformly non-square.

Example 5. Let $X$ be $\mathbb{R}^{2}$ endowed with the $\ell_{\infty}-\ell_{1}$ norm defined by

$$
\|x\|=\left\|\left(x_{1}, x_{2}\right)\right\|= \begin{cases}\max \left\{\left|x_{1}\right|,\left|x_{2}\right|\right\}, & x_{1} x_{2} \geq 0 \\ \left|x_{1}\right|+\left|x_{2}\right|, & x_{1} x_{2}<0\end{cases}
$$

Assume that $x=(1,1), y=(1,0) \in S_{X}$; then $x-y=(0,1) \in S_{X}$. Then,

$$
M_{3}(X) \geq \frac{\|x+y\|^{\frac{1}{2}}+\|2 x-y\|^{\frac{1}{2}}}{2}=\sqrt{2}
$$

and then we have

$$
M_{3}(X) \leq \frac{(\|x\|+\|y\|)^{\frac{1}{2}}+(\|x\|+\|x-y\|)^{\frac{1}{2}}}{2}=\sqrt{2} .
$$


Therefore,

$$
M_{3}(X)=\sqrt{2} .
$$

\section{Conclusions}

In light of the asymmetric geometric constant $H(X)$ proposed by Gao and the Baronti type constant $A_{2}(X)$, we intend to introduce a new geometric constant $M_{1}(X)$, which measures the lengths of two midlines of a triangle with one vertex at the center of the unit ball and the other on the unit sphere. In this paper, we manage to show several geometric properties, such as rotundity and the uniform nonsquare property, via characterizing its relationships with a variety of other basic geometric constants. Moreover, we provide a study of the quadratic form and subduplicate form of this constant, $M_{2}(X)$ and $M_{3}(X)$, respectively, by means of exploration of their relations with some well-known geometric constants. However, there are still plenty of interesting problems that await discussion. How can $M_{1}(X), M_{2}(X)$ and $M_{3}(X)$ be utilized to characterize more geometric properties? More results will be presented in our future research for the reader interested in going further in geometric constants of a Banach space.

Author Contributions: Writing—original draft preparation, B.C., Z.Y., Q.L. and Y.L.; writing—review and editing, B.C., Z.Y., Q.L. and Y.L. All authors have read and agreed to the published version of the manuscript.

Funding: This research received no external funding.

Institutional Review Board Statement: Not applicable.

Informed Consent Statement: Not applicable.

Acknowledgments: The authors are immensely grateful for the insightful and invaluable suggestions given by the reviewers who contributed to the corresponding revisions of the manuscript.

Conflicts of Interest: The authors declare no conflict of interest.

\section{References}

1. Baronti, M.; Casini, E.; Papini, P.L. Triangles inscribed in a semicircle, in Minkowski planes. J. Math. Anal. Appl. 2000, 252, $124-146$. [CrossRef]

2. Alonso, J.; Llorens-Fuster, E. Geometric mean and triangles inscribed in a semicircle in banach spaces. J. Math. Anal. Appl. 2008, 340, 1271-1283. [CrossRef]

3. Gao, J. Normal hexagon and more general Banach spaces with uniform normal structure. J. Math. 2000, 20, 241-248.

4. Chen, B.; Liu, Q.; Li, Y. Inscribed triangles in the unit sphere and a new class of geometric constants. Symmetry 2022, $14,72$. [CrossRef]

5. Yang, C.; Wang, F. On a new geometric constant related to the von Neumann-Jordan constant. J. Math. Anal. Appl. 2006, 324, 555-565. [CrossRef]

6. Lindenstrauss, J. On the modulus of smoothness and divergent series in Banach spaces. Mich. Math. J. 1963, 10, $241-252$. [CrossRef]

7. Gao, J. The uniform degree of the unit ball of a Banach space I. Nanjing Daxue Xuebao 1982, 1, 14-28. (In Chinese)

8. Gao, J.; Lau, K.S. On two classes Banach spaces with uniform normal structure. Stud. Math. 1991, 99, 41-56. [CrossRef]

9. Clarkson, J.A. The von Neumann-Jordan constant for the Lebesgue space. Ann. Math. 1937, 38, 114-115. [CrossRef]

10. Alonso, J.; Martin, P.; Papini, P.L. Wheeling around von Neumann-Jordan constant in Banach spaces. Stud. Math. 2008, 188, 135-150. [CrossRef]

11. Kato, M.; Maligranda, L.; Takahashi, Y. On James, Jordan-von Neumann constants and the normal structure coefficient of Banach spaces. Stud. Math. 2001, 144, 275-295. [CrossRef]

12. Takahashi, Y. Some geometric constants of Banach spaces-A unified approach. In Banach and Function Spaces II; Kato, M., Maligranda, L., Eds.; Yokohama Publishers: Yokohama, Japan, 2007; pp. 191-220.

13. Alsina, C.; Tomás, M.S. Orthocenters in real normed spaces and the Euler line. Aequ. Math. 2004, 67, 180-187. [CrossRef]

14. García-Falset, J.; Llorens-Fuster, E.; Mazcuńan-Navarro, E.M. Uniformly nonsquare Banach spaces have the fixed point property for nonexpansive mappings. J. Funct. Anal. 2006, 233, 494-514. [CrossRef] 